\title{
Editorial: Machine Learning and Intelligent Wireless Communications (MLICOM 2019)
}

\author{
Xiangping Bryce Zhai ${ }^{1} \cdot$ Congduan $\mathrm{Li}^{2} \cdot \mathrm{Kai}^{\mathrm{Liu}}{ }^{3}$ \\ Published online: 10 July 2020 \\ (C) Springer Science+Business Media, LLC, part of Springer Nature 2020
}

\section{Editorial}

With the advent of the fourth industrial revolution and the fast development of virtual/augmented reality, the number of high quality wireless services is exponentially increasing. According to the prediction of Cisco VNI Mobile Forecast 2017, global mobile data traffic will increase sevenfold between 2016 and 2021, and the speed of mobile network connections will increase threefold to 20.4 megabits per second (Mbps) by 2021. Hence, there is still a big gap between the future requirements and current communications technologies, even using $4 \mathrm{G} / 5 \mathrm{G}$. This motivates the researchers to improve the system performance by integrating the limited wireless resources with some intelligent algorithms/schemes.

As an emerging discipline, machine learning is a subfield of computer science that evolves pattern recognition and computational learning theory in artificial intelligence, which can be further used to make predictions on complicated scenarios. In communication systems, the previous/current radio situations and communication paradigms should be well considered to obtain a high quality of service, such as high communication rate, energy saving, consumption reducing, and the robust of communications for high mobility especially in large scale networks. We hope that integrating machine learning algorithms into communication systems will improve the systems to be smarter, more intelligent, and more efficient. Thus, this is a good time to call for intelligent solutions that can be

Xiangping Bryce Zhai

blueicezhaixp@nuaa.edu.cn

1 College of Computer Science and Technology, Nanjing University of Aeronautics and Astronautics, and Key Laboratory of Safety-Critical Software (Nanjing University of Aeronautics and Astronautics), Ministry of Industry and Information Technology, Nanjing, China

2 School of Electronics and Communications Engineering at the Sun Yat-sen University, Shenzhen, China

3 College of Computer Science, Chongqing University, Chongqing, China

applied to future mobile communications and networks. We aim to explore intelligent/machine-learning algorithms for mobile communication networking systems. Expected contributions call upon a wide range of novel modeling as well as algorithmic and computational frameworks related to intelligent optimization or machine learning.

This special issue features eleven selected papers with high quality. The first article with the title "Clustered-NOMA Based Resource Allocation in Wireless Powered Communication Networks" authored by Zhenyu Na, Yue Liu, Jun Wang, Mingxiang Guan, and Zihe Gao, proposed a clustered-Non-Orthogonal Multiple Access based wireless powered communication model. The authors adopted a terminal clustering scheme based on maximizing the sum of channel condition difference between terminals, and a time-sharing mechanism to decode information.

Simulation results demonstrated both the maximization of the sum rate in the uplink and the enhancement of the fairness between terminals.

The second article titled "FedMEC: Improving Efficiency of Differentially Private Federated Learning via Mobile Edge Computing" from Jiale Zhang, Yanchao Zhao, Junyu Wang, and Bing Chen, aimed to reduce the heavy computation cost of deep neural network training on edge devices and to provide strong privacy guarantees. The authors proposed a federated learning framework to integrate model partition technique and differential privacy simultaneously. Furthermore, they prevented the privacy leakage from the local model parameters. Experiments results validated the effectiveness and practicality.

In the next article with the title "Design of Extreme Learning Machine with Smoothed L0 Regularization" authored by Cuili Yang, Kaizhe Nie, Junfei Qiao, and Bing $\mathrm{Li}$, the authors studied the extreme learning machine with smoothed L0 regularizer to improve network compactness. They investigated both the weak convergence and strong convergence. Compared to other existing extreme learning machines, the proposed algorithm achieved better performance in terms of estimation accuracy and network sparsity. 
The fourth article titled "Gesture Recognition through sEMG with Wearable Device based on Deep Learning" from Shu Shen, Kang Gu, Xinrong Chen, Caixia Lv, and Ruchuan Wang, designed a surface electromyography signal classification gesture recognition system using deep learning based on wearable devices. The system consisted of surface electromyography acquisition devices, preprocessing and surface electromyography gesture recognition model based on convolutional neural networks. Compared to Linear Discriminant Analysis, Support Vector Machine, and Long Short Term MemoryConvolutional Neural Networks and Random Forests, the accuracy was improved.

The fifth article titled "Topology Sensing of Wireless Networks Based on Hawkes Process" from Zitong Liu, Jiachen Sun, Feng Shen, Zheng Wang, and Gao Li, investigated topology sensing with unreliable information caused by imperfect channels in wireless heterogeneous networks. The authors proposed a wireless channel-oriented topology sensing scheme based on Hawkes process. In addition, they analyzed the performance under various parameter configurations. Simulations demonstrated the effective scheme for the imperfect channels.

In the next article with the title "Cost Sensitive Learning Based HEVC Screen Content Intra Coding for Mobile Devices" authored by Yuanyuan Xu, and Kun Zhu, the authors proposed a flexible screen content intra coding scheme, to trade between encoding complexity and rate-distortion performance degradation via cost sensitive learning. They designed binary classifiers and adopted the cost-proportionate weighted sampling. Experimental results showed the effective flexible coding policy offline.

The seventh article titled "Vehicular Fog Computing Enabled Real-time Collision Warning via Trajectory Calibration" from Xincao Xu, Kai Liu, Ke Xiao, Liang Feng, Zhou Wu, and Songtao Guo, aimed to support effective and real-time collision warning in a vehicular fog computing architecture. The authors proposed a trajectory calibration based collision warning algorithm along with tailored communication protocols. Then, they designed a packet loss detection mechanism. Simulation results demonstrated the effectiveness in terms of the highest precision and recall in a wide range of scenarios.

The eighth article with the title "A Design of SDR-based Pseudo-Analog Wireless Video Transmission System" authored by Xiao-Wei Tang, and Xin-Lin Huang, presented a novel design of software radio platform-based pseudo-ana$\log$ wireless video transceiver. The authors proved that the analog method can achieve the optimal performance based on the rate-distortion theory. Then, they introduced implementation details and analyzed the performance of the designed transceiver. Simulation and experiment results showed the designed effective system.

The ninth article titled "Root Cause Analysis for Selforganizing Cellular Network: An Active Learning Approach" from Meng Chen, Kun Zhu, and Bing Chen, proposed an active learning based fault diagnosis scheme to achieve an effective classier and dramatically reduce the number of needed labeled instances in self-organizing networks. The authors applied uncertainty sampling as the query strategy. They constructed a long term evolution system level simulator to verify the effective scheme.

In the next article with the title "Task Offloading Scheme Based on Improved Contract Net Protocol and Beetle Antennae Search Algorithm in Fog Computing Networks" from Xujie Li, Zhennan Zang, Fei Shen, and Ying Sun, the authors proposed a task offloading scheme based on improved contract net protocol and beetle antennae search algorithm in fog computing networks. They obtained the information from the fog nodes to allocate the sub-tasks. Simulation results validated the efficiency of the proposed scheme compared with other algorithms.

The last article titled "Energy-efficient Resource Optimization in Green Cognitive Internet of Things" from Xin Liu, Ying Li, Xueyan Zhang, Weidang Lu, and Mudi Xiong, proposed a green Cognitive Internet of Things to collect the radio frequency energy of primary user by using energy harvesting. Then, the authors guaranteed the energy balance to prolong the life of system with the help of the spectrum sensing and energy harvesting. Simulation results indicated the existence of the optimal solution and the outstanding performance.

Acknowledgements The guest editors are thankful to our reviewers for their effort in reviewing the manuscripts. We also thank the Edit-in-Chief, Dr. Imrich Chlamtac for his supportive guidance during the entire process. The special issue is sponsored in part by National Natural Science Foundation of China (No. 61701231, No. 61901534), and the Foundation of Key Laboratory of Safety-Critical Software (Nanjing University of Aeronautics and Astronautics), Ministry of Industry and Information Technology (No. NJ2020022), in part by the Guangdong Basic and Applied Basic Research Foundation under Key Project (No. 2019B1515120032), in part by the Science, Technology and Innovation Commission of Shenzhen Municipality (No. JCYJ20190807155617099).

Publisher's note Springer Nature remains neutral with regard to jurisdictional claims in published maps and institutional affiliations. 


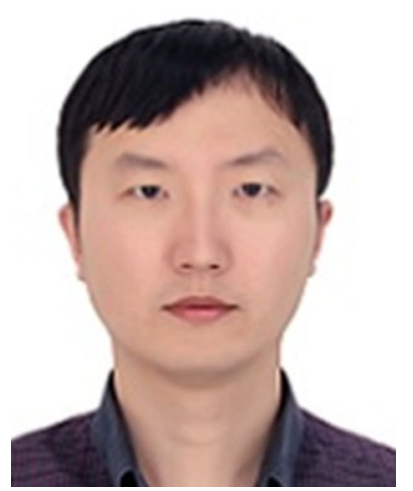

Xiangping Bryce Zhai (M'15, IEEE) received the B.Eng. degree in Computer Science and Technology from Shandong University in 2006, and the $\mathrm{Ph}$.D. degree in Computer Science from City University of Hong Kong in 2013. Previously, he was a Postdoctoral Fellow at the City University of Hong Kong. He is currently an Associate Professor of the College of Computer Science and Technology, Nanjing University of Aeronautics and Astronautics, and Key Laboratory of Safety-Critical Software (Nanjing University of Aeronautics and Astronautics), Ministry of Industry and Information Technology, China. His research interests are in the area of Internet of Things, power control, edge computing, resource optimization and unmanned aerial vehicle. He has been actively involved in organizing and chairing sessions, and has served as reviewer for several journals and TPC for several international conferences.

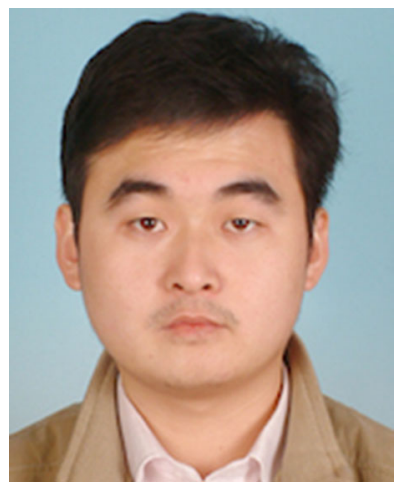

Congduan Li (S'11-M'15, IEEE) received the B.S. degree from the University of Science and Technology Beijing, China, in 2008, the M.S. degree from Northern Arizona University, Flagstaff, AZ, USA in 2011, and Ph.D. degree from Drexel University, Philadelphia, PA, USA in 2015, respectively, all in Electrical Engineering. From Oct 2015 to Aug 2018, he was a postdoctoral research fellow in the Institute of Network Coding at the Chinese University of Hong Kong and the Department of Computer Science at City University of Hong Kong. He is currently an Associate Professor in School of Electronics and Communications Engineering at the Sun Yatsen University. His research interests lie in information theory, network coding, wireless communications, and machine learning, etc.

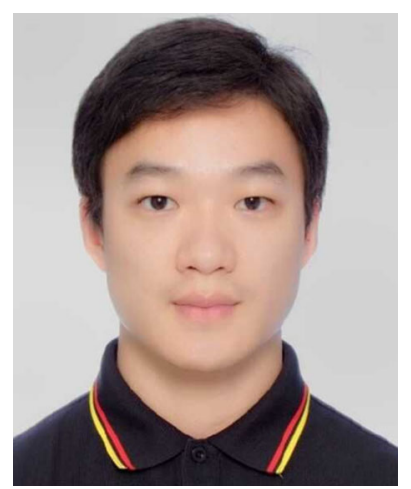

Kai Liu (S'07-M'12-SM'19,

IEEE) received his Ph.D. Degree in Computer Science from the City University of Hong Kong in 2011. From December 2010 to May 2011, he was a Visiting Scholar with the Department of Computer Science, University of Virginia, USA. From 2011 to 2014, he was a Postdoctoral Fellow with Singapore Nanyang Technological University, City University of Hong Kong, and Hong Kong Baptist University. $\mathrm{He}$ is currently a Professor with the College of Computer Science, Chongqing University, China. His research interests include Internet of Vehicles, Mobile Computing and Pervasive Computing. 